\title{
A comparison of the effects of midazolam, propofol and dexmedetomidine on the antioxidant system: A randomized trial
}

\author{
CHAO HAN $^{1 *}$, WEILIANG DING ${ }^{2 *}$, WENJIE JIANG $^{1 *}$, YU CHEN $^{3}$, DONGYUAN HANG $^{1}$, \\ DAMING GU ${ }^{1}$, GUOJUN JIANG ${ }^{4}$, YONGFEI TAN ${ }^{4}$, ZHIJUN GE $^{5}$ and TIELIANG MA ${ }^{2}$ \\ ${ }^{1}$ Department of Anesthesiology; ${ }^{2}$ Central Laboratory, The Affiliated Yixing Hospital of Jiangsu University, Yixing; \\ ${ }^{3}$ Department of Anesthesiology, Jiangsu Province Hospital, Nanjing; Departments of ${ }^{4}$ Critical Care Medicine and \\ ${ }^{5}$ Cardiac and Thoracic Surgery, The Affiliated Yixing Hospital of Jiangsu University, Yixing, Jiangsu 214200, P.R. China
}

Received July 17, 2014; Accepted March 18, 2015

DOI: $10.3892 / \mathrm{etm} .2015 .2410$

\begin{abstract}
Previous studies on the antioxidant activity of sedatives have predominantly been in vitro investigations that are lacking clinical data, resulting in conclusion without cogency. The aim of the present prospective, randomized study was to use single sedative drugs for anesthesia induction to compare their antioxidant properties. The effects on the antioxidant system of midazolam, propofol and dexmedetomidine were assessed using oxidative stress indicators and micronuclei (MN). Forty-nine patients undergoing esophageal cancer radical prostatectomy were selected. Midazolam, propofol and dexmedetomidine were used to induce anesthesia. Venous blood samples were obtained prior to and at 2 and at $24 \mathrm{~h}$ after surgery, and oxidative stress indicators were detected using the appropriate kits. The cytokinesis-block micronucleus cytome assay was executed and the frequencies of $\mathrm{MN}$, nucleoplasmic bridges and nuclear buds were examined. It was found that the use of the three sedatives, respectively, led to a marked increase in the levels of free radical indicators at $2 \mathrm{~h}$ after surgery, which then decreased at $24 \mathrm{~h}$ after surgery. Furthermore, lower levels of oxidative stress were found following the use of propofol and dexmedetomidine compared with those following midazolam administration, and similar results were obtained regarding
\end{abstract}

Correspondence to: Dr Tieliang Ma, Central Laboratory, The Affiliated Yixing Hospital of Jiangsu University, 75 Tongzhenguan Road, Yixing, Jiangsu 214200, P.R. China

E-mail: matieliang@foxmail.com

Professor Zhijun Ge, Department of Cardiac and Thoracic Surgery, The Affiliated Yixing Hospital of Jiangsu University, 75 Tongzhenguan Road, Yixing, Jiangsu 214200, P.R. China

E-mail: staff789@yxph.com

${ }^{*}$ Contributed equally

Key words: midazolam, propofol, dexmedetomidine, oxidative stress, micronucleus the level of MN. It is suggested that propofol and dexmedetomidine exhibit a superior antioxidant function to midazolam.

\section{Introduction}

Oxidative stress is defined as a state in which the production of reactive oxygen species (ROS) overcomes the endogenous antioxidant defenses of the host, which leads to lipid, protein and DNA damage. Lipid peroxidation, which is the main method by which free radicals induce cellular damage, can cause decreases in membrane fluidity and permeability, and membrane protein denaturation. Oxidative stress can also lead to DNA damage, which is associated with chromosome aberrations and micronucleus formation (1). Micronuclei (MN) result from chromosomal fragments or lagging chromosomes during cell division, which are not included in the main nucleus, existing independently in the cytoplasm. MN are a main biological marker of chromosomal instability (2).

An association has been proposed between increased oxidative stress and poor outcomes in the critically ill, suggesting a potential role for antioxidant strategies in the intensive care unit (ICU) (3). Limiting levels of oxidative stress may prevent cellular death, decrease inflammation and reduce morbidity and mortality rates (4); therefore, the antioxidant properties of sedative drugs should be taken into consideration as an important part of ICU remedy strategies, particularly when mechanical ventilation is required. Previously, Kang et al (5) classified the antioxidant potential of various drugs used in the perioperative period; however, to the best of our knowledge, a comparison of the effects on the antioxidant system of dexmedetomidine, propofol and midazolam, which are most frequently used in the ICU, has yet to be documented. The aim of the present study, therefore, was to investigate the antioxidant properties of the three sedative drugs.

Numerous clinical studies have revealed the involvement of ROS in severe invasive surgeries, such as thoracotomy, open-heart surgery and organ transplantation (6-10). In the present study, a clinical model was designed in which a single sedative drug was used for anesthesia induction and maintenance to achieve a definite depth of anesthesia in esophageal 
cancer radical prostatectomy. Venous blood samples obtained prior to surgery (T0) and at $2 \mathrm{~h}$ (T1) and $24 \mathrm{~h}$ (T2) after surgery were tested to confirm the levels of ROS generation (superoxide anion, hydrogen peroxide and hydroxyl radical), the activity of endogenous antioxidant enzymes [superoxide dismutase (SOD), glutathione peroxidase (GSH-Px) and catalase (CAT)] and the levels of lipid peroxidation product [malondialdehyde (MDA)] $(11,12)$. DNA damage was detected using the cytokinesis-block micronucleus cytome assay (CBMN) (2).

\section{Materials and methods}

Study subjects. The study was approved by the Ethics Committee of the Affiliated Yixing Hospital of Jiangsu University (Yixing, China) and written informed consent was obtained from each of the participants. Forty-nine patients of Society of Anesthesiologists physical status I-II, who were undergoing esophageal cancer radical prostatectomy, were randomly divided into three groups: Midazolam (M group, $\mathrm{n}=16$ ), propofol ( $\mathrm{P}$ group, $\mathrm{n}=16$ ) and dexmedetomidine (D group, $n=17$ ). Patients with liver or renal dysfunction or hemostatic disorders were excluded. The administration of antioxidants, including vitamins $\mathrm{C}$ and $\mathrm{E}$, edaravone, ebselen, resveratrol and other Chinese herbs that have been suggested to possess antioxidant properties, was not permitted during the perioperative period.

Anesthesia. All patients received $0.5 \mathrm{mg}$ atropine intramuscularly as premedication $30 \mathrm{~min}$ prior to entering the operating theatre. In the $\mathbf{M}$ group, anesthesia induction was performed through the intravenous injection of midazolam (0.3 $\mathrm{mg} / \mathrm{kg})$, fentanyl $(5 \mu \mathrm{g} / \mathrm{kg})$ and vecuronium bromide $(0.15 \mathrm{mg} / \mathrm{kg})$, and maintained by intravenous infusion of midazolam $(0.5-1.5 \mu \mathrm{g} / \mathrm{kg} / \mathrm{min})$ and fentanyl $(0.05 \mu \mathrm{g} / \mathrm{kg} / \mathrm{min})$. In the $\mathrm{P}$ group, anesthesia was induced by intravenous injection of propofol $(1 \mathrm{mg} / \mathrm{kg})$, fentanyl $(5 \mu \mathrm{g} / \mathrm{kg})$ and vecuronium bromide $(0.15 \mathrm{mg} / \mathrm{kg})$, and maintained by intravenous infusion of propofol (100-200 $\mu \mathrm{g} / \mathrm{kg} / \mathrm{min})$ and fentanyl $(0.05 \mu \mathrm{g} / \mathrm{kg} / \mathrm{min})$. In the D group, anesthesia was induced by intravenous injection of dexmedetomidine $(0.5 \mu \mathrm{g} / \mathrm{kg})$, fentanyl $(5 \mu \mathrm{g} / \mathrm{kg})$ and vecuronium bromide $(0.15 \mathrm{mg} / \mathrm{kg})$, and maintained by intravenous infusion of dexmedetomidine $(0.04-0.08 \mu \mathrm{g} / \mathrm{kg} / \mathrm{min})$ and fentanyl $(0.05 \mu \mathrm{g} / \mathrm{kg} / \mathrm{min})$. All patients received tracheal intubation and underwent mechanical ventilation with $100 \%$ oxygen (tidal volume, $8-10 \mathrm{ml} / \mathrm{kg}$; respiratory frequency, $10-14 / \mathrm{min}$ ) with the aim of achieving an end-tidal carbon dioxide level of $38-40 \mathrm{mmHg}$ during the surgical procedure. An extra fentanyl dose $(5 \mu \mathrm{g} / \mathrm{kg})$ was administered to the patients 3 min before the surgery. The Cerebral State Index (CSI) monitor, an electroencephalogram-based monitor that has a similar performance to the Bispectral Index in terms of predicting the clinical state of the patient assessed by the Observer's Assessment of Alertness/Sedation scale (9), was used to monitor the depth of anesthesia during the surgery. The CSI value was maintained at $\sim 50$ by adjusting the dose of the sedative drugs. Vasopressors were administered to regulate mean arterial pressure (MAP) at basal level. The vasoactive agents and corresponding dose ranges were dopamine $(6-30 \mu \mathrm{g} / \mathrm{kg} / \mathrm{min})$ and nitroglycerin $(1-5 \mu \mathrm{g} / \mathrm{kg} / \mathrm{min})$.
Post-operative management. Following surgery, the patients were admitted to the Post-Anesthesia Care Unit (PACU) for monitoring. In addition to antimicrobial, expectorant, antacid and nutrition therapy, intravenous morphine infusion $(15-25 \mu \mathrm{g} / \mathrm{kg} / \mathrm{h})$ was used for postoperative analgesia. The patients were extubated when there was no indication of bleeding and the patient was observed to be alert, cardiovascularly stable and normothermic, with an arterial oxygen tension of $>74 \mathrm{mmHg}$, an inspired oxygen concentration of $<40 \%$ and a positive end-expiratory pressure of $<5 \mathrm{cmH}_{2} \mathrm{O}$. Prior to the patients being discharged from the ward, three criteria had to be met: i) Consciousness; ii) spontaneous breathing without an endotracheal tube; and iii) stable hemodynamics without vasoactive drug administration.

Study protocol. The MAP, heart rate (HR), oxygen saturation and fluid volume were continuously monitored during the surgical procedure and in the PACU. The time until recovery of consciousness, tracheal extubation time and length of PACU stay were recorded. Venous blood samples were obtained prior to the surgery (T0) and at $2 \mathrm{~h}$ (T1) and $24 \mathrm{~h}$ (T2) after the surgery. The samples were then centrifuged at $1,000 \mathrm{x} g$ for $15 \mathrm{~min}$ at room temperature and the serum samples were stored at $-80^{\circ} \mathrm{C}$ until analysis.

Biochemical analysis. In this study, oxidative stress indicators were divided into three categories: Free radical indicators (superoxide anion, hydrogen peroxide and hydroxyl radical), free radical damage indicators (MDA) and endogenous antioxidant indicators (SOD, GSH-Px and CAT). All procedures were performed according to the instructions provided with the kits from Jiancheng Bioengineering Research Institute (Nanjing, China): Superoxide anion, A052; hydrogen peroxide, A064-1; hydroxyl radical, A018; MDA, A003-1; SOD, A001-1; GSH-Px, A005; and CAT, A007-2.

$C B M N$. Blood samples were drawn from the subjects. A sample of whole blood $(0.5 \mathrm{ml})$ was added to $4.5 \mathrm{ml}$ RPMI-1640 culture medium and phytohemagglutinin, which was required for lymphocyte stimulation. Cytochalasin B (6 $\mu \mathrm{g} / \mathrm{ml}$; Sigma-Aldrich, St. Louis, MO, USA) was added after $44 \mathrm{~h}$ of culture to block cytokinesis, which facilitated the identification of lymphocytes that had divided in culture. As such, cells that had undergone the first mitotic division were recognized as binucleated cells and were selectively screened for the presence of MN, nucleoplasmic bridges (NPBs) and nuclear buds (NBUDs) (7). Cell harvesting, hypotonic treatment, fixation and slide preparation were performed following standard procedures. The presence of MN was scored blindly in 1,000 binucleated cells, in accordance with standard criteria, and the frequency was expressed as the number of binucleated cells containing one or more MN/NPBs/NBUDs per 1,000 cells (2).

Statistical analysis. Categorical variables are presented as percentages and continuous variables are expressed as the mean \pm standard deviation. Differences between variables were analyzed using the Student's t-test (continuous variables) and the $\chi^{2}$ test (categorical variables). Data analysis was performed using SPSS for Windows software version 14.0 (SPSS, Inc., 
Table I. Demographics and clinical characteristics.

\begin{tabular}{lccc}
\hline Characteristic & M group & P group & D group \\
\hline Age (years) & $70.4 \pm 4.9$ & $72.3 \pm 4.6$ & $68.7 \pm 5.2$ \\
Gender (male/female) & $12 / 4$ & $12 / 4$ & $12 / 5$ \\
Weight (kg) & $61.17 \pm 9.52$ & $57.43 \pm 7.25$ & $59.48 \pm 8.83$ \\
Surgery duration (h) & $2.4 \pm 0.4$ & $2.2 \pm 0.3$ & $2.1 \pm 0.3$ \\
Blood loss (ml) & $249 \pm 45$ & $228 \pm 36$ & $242 \pm 34$ \\
Fluid balance in surgery (ml) & $1,356 \pm 74$ & $1,418 \pm 88$ & $1,462 \pm 93$ \\
Use of vasoactive agents (n) & 6 & 5 & 5 \\
Intraoperative fentanyl requirement (mg) & $0.68 \pm 0.06$ & $0.63 \pm 0.04$ & $0.67 \pm 0.05$ \\
Postoperative morphine requirement (mg) & $64.6 \pm 4.7$ & $62.3 \pm 3.6$ & $1.2 \pm 0.4^{\mathrm{a}}$ \\
Time until recovery of consciousness (h) & $2.5 \pm 0.8$ & $2.8 \pm 1.2^{\mathrm{a}}$ & $1.6 \pm 0.5^{\mathrm{a}}$ \\
Tracheal extubation time (h) & $4.6 \pm 1.3$ & $41.7 \pm 4.2$ & $3.0 \pm 0.9^{\mathrm{a}}$ \\
Length of stay in the PACU (h) & $43.4 \pm 4.7$ & $43.1 \pm 5.1$ \\
\hline
\end{tabular}

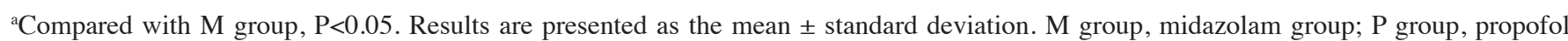
group; D group, dexmedetomidine group; PACU, Post-Anesthesia Care Unit.

Chicago, IL, USA). $\mathrm{P}<0.05$ was considered to indicate a statistically significant difference.

\section{Results}

Patient characteristics. The clinical characteristics of the patients are summarized in Table I. The groups were similar in terms of age, gender, weight, surgery duration, blood loss, fluid volume, use of vasoactive agents, dose of intraoperative fentanyl, dose of postoperative morphine and length of stay in the PACU; however, the time until consciousness was recovered and tracheal extubation time in the $M$ group were significantly longer than the results for the other two groups $(\mathrm{P}<0.05)$. All patients in the perioperative period were in steady state, and no postoperative complications were observed. The blood pressure, HR and other vital signs of the patients of the three groups exhibited no significant differences $(\mathrm{P}>0.05)$ (Fig. 1).

Levels of free radical, free radical damage and endogenous antioxidant indicators. To assess the levels of oxidative stress, free radical indicators (superoxide anion, hydrogen peroxide and hydroxyl radical) were detected in the plasma at different time-points. The results showed that the levels of the three indicators were increased significantly $2 \mathrm{~h}$ after surgery, but then returned to levels approaching those prior to the surgery. Furthermore, compared with the $\mathrm{M}$ group, the levels of the three indicators were significantly lower in the $\mathrm{P}$ and $\mathrm{D}$ groups (Table II).

The levels of MDA showed similar trends to the free radical indicators; however, the level of MDA in the D group was significantly lower than that in the $\mathrm{P}$ group at T2, while the levels in the $\mathrm{P}$ and $\mathrm{D}$ groups were both lower than that observed in the $\mathrm{M}$ group (Table III).

With regard to the endogenous antioxidant indicators (SOD and GSH-Px), the levels at T1 were found to be lower than the presurgery values in the three groups. Furthermore,
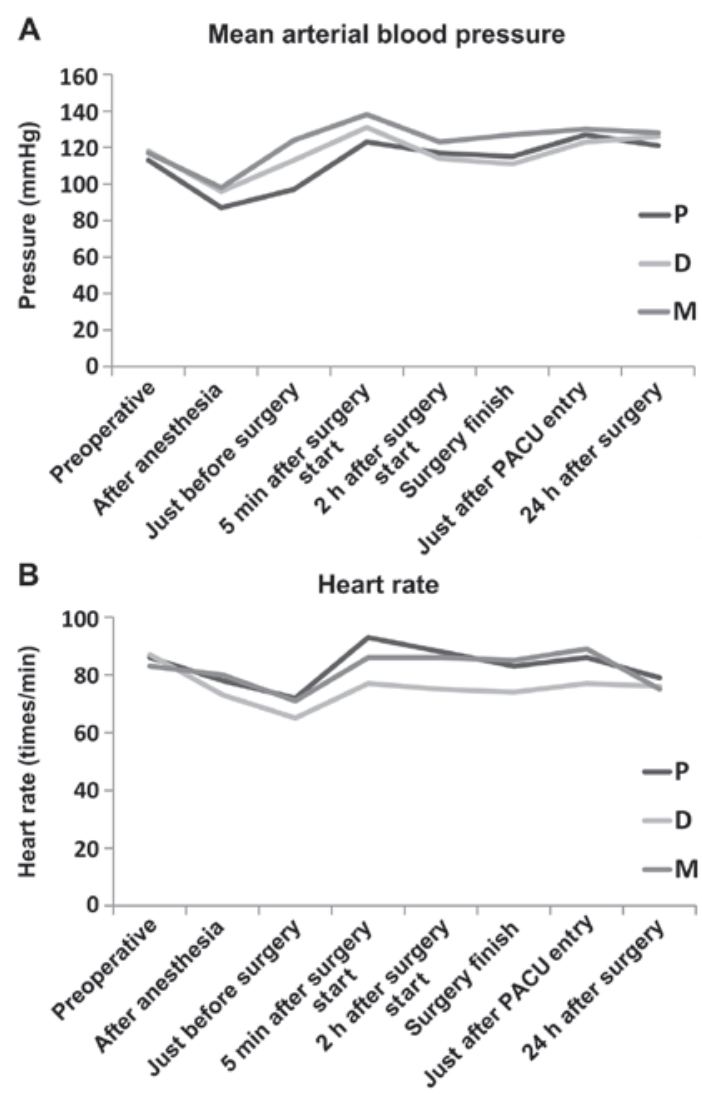

Figure 1. Results for the (A) mean arterial blood pressure and (B) heart rate $(\mathrm{B})$ at different time-points in the three groups. $\mathrm{M}$ group, midazolam group; P group, propofol group; D group, dexmedetomidine group; PACU, Post-Anesthesia Care Unit.

the levels of SOD and GSH-Px were significantly higher in the $\mathrm{P}$ and $\mathrm{D}$ groups compared with those in the M group. Notably, the level of CAT did not exhibit any significant changes among the three groups (Table IV). In these cases, it was concluded that more desirable effects were found in the P and D groups. 
Table II. Levels of superoxide anions, hydroxyl radicals and hydrogen peroxide.

\begin{tabular}{|c|c|c|c|}
\hline Indicator & T0 & $\mathrm{T} 1$ & $\mathrm{~T} 2$ \\
\hline \multicolumn{4}{|c|}{ Superoxide anion (U/l) } \\
\hline M group & $109.0 \pm 24.3$ & $180.9 \pm 45.7^{\mathrm{a}}$ & $126.7 \pm 41.9^{b}$ \\
\hline$P$ group & $103.7 \pm 20.3$ & $144.6 \pm 34.2^{\mathrm{a}, \mathrm{c}}$ & $117.3 \pm 32.3^{\mathrm{b}, \mathrm{c}}$ \\
\hline D group & $112.8 \pm 31.1$ & $160.2 \pm 37.7^{\mathrm{a}, \mathrm{c}}$ & $113.0 \pm 39.0^{\mathrm{b}, \mathrm{c}}$ \\
\hline \multicolumn{4}{|c|}{ Hydroxyl radical (U/ml) } \\
\hline M group & $336.5 \pm 83.8$ & $580.3 \pm 91.4^{\mathrm{a}}$ & $379.5 \pm 78.3^{\mathrm{b}}$ \\
\hline P group & $339.7 \pm 56.6$ & $450.7 \pm 82.6^{\mathrm{a}, \mathrm{c}}$ & $353.4 \pm 82.9^{\mathrm{b}, \mathrm{c}}$ \\
\hline D group & $347.3 \pm 84.2$ & $419.2 \pm 77.2^{\mathrm{a}, \mathrm{c}}$ & $363.4 \pm 79.1^{\mathrm{b}, \mathrm{c}}$ \\
\hline \multicolumn{4}{|c|}{ Hydrogen peroxide (mmol/l) } \\
\hline M group & $35.4 \pm 9.5$ & $78.1 \pm 12.8^{\mathrm{a}}$ & $38.1 \pm 8.3^{\mathrm{b}}$ \\
\hline P group & $33.6 \pm 8.3$ & $58.0 \pm 16.1^{\mathrm{a}, \mathrm{c}}$ & $36.2 \pm 7.9^{\mathrm{b}, \mathrm{c}}$ \\
\hline D group & $35.4 \pm 8.1$ & $55.3 \pm 10.8^{\mathrm{a}, \mathrm{c}}$ & $35.7 \pm 6.4^{\mathrm{b}, \mathrm{c}}$ \\
\hline
\end{tabular}

${ }^{\mathrm{a} C}$ Compared with $\mathrm{T} 0, \mathrm{P}<0.05$; ${ }^{\mathrm{b}}$ compared with $\mathrm{T} 1, \mathrm{P}<0.05$; ${ }^{\mathrm{c}}$ compared with $\mathrm{M}$ group, $\mathrm{P}<0.05$. Results are presented as the mean \pm standard deviation. M group, midazolam group; P group, propofol group; D group, dexmedetomidine group.

Table III. Levels of malondialdehyde.

\begin{tabular}{lccc}
\hline Group & T0 & T1 & T2 \\
\hline M & $5.1 \pm 1.0$ & $9.5 \pm 2.4^{\mathrm{a}}$ & $7.3 \pm 1.4^{\mathrm{b}}$ \\
P & $5.2 \pm 0.8$ & $8.3 \pm 0.9^{\mathrm{a}, \mathrm{c}}$ & $6.8 \pm 1.1^{\mathrm{b}, \mathrm{c}}$ \\
$\mathrm{D}$ & $5.1 \pm 0.9$ & $7.9 \pm 1.3^{\mathrm{a}, \mathrm{c}}$ & $5.3 \pm 1.2^{\mathrm{b}, \mathrm{c}, \mathrm{d}}$
\end{tabular}

${ }^{\mathrm{a} C}$ Compared with $\mathrm{T} 0, \mathrm{P}<0.05$; ${ }^{\mathrm{b}}$ compared with $\mathrm{T} 1, \mathrm{P}<0.05$; ${ }^{\mathrm{c}}$ compared with $\mathrm{M}$ group, $\mathrm{P}<0.05$; ${ }^{\mathrm{d}}$ compared with $\mathrm{P}$ group, $\mathrm{P}<0.05$. Results are presented as the mean \pm standard deviation. M group, midazolam group; P group, propofol group; D group, dexmedetomidine group.

Chromosomal instability of lymphocytes in the patients. In order to examine the oxidative damage of the lymphocytes from the blood samples, the CBMN assay was utilized to detect the frequencies of MN, NPBs and NBUDs at T0, T1 and T2. It was found that the micronucleus and NPB frequencies exhibited a time-dependent effect, with increases at $\mathrm{T} 1$ and decreases at T2; however, no significant differences among the three time-points were found for the NBUD frequency. It was further noted that the micronucleus frequency was significantly lower in the $\mathrm{P}$ and $\mathrm{D}$ groups than that in the $\mathrm{M}$ group at T1 and T2 (Table V). We therefore speculated that propofol and dexmedetomidine caused less damage than midazolam.

\section{Discussion}

Although a previous in vitro study demonstrated that the majority of sedative drugs exhibit antioxidant activity (5), the application of sedative drugs has, for a long time, been mainly based on the pharmacology and pharmacokinetic properties of the drugs, without considering their antioxidant activity and effect on progress and prognosis. Previous studies on the antioxidant activity of sedatives are predominantly in vitro investigations (5) that are lacking clinical data, resulting in conclusion without cogency. In the present study, a specific major surgery (esophageal cancer radical prostatectomy) and standardized remedy strategies were selected as a clinical model of oxidative stress. Various sedative drugs were then used in general anesthesia; the changes in the levels of oxidative stress indicators were detected and the antioxidant activities of the sedatives were evaluated.

A number of different mechanisms can lead to the increase in free radical levels caused by surgical trauma: Mitochondrial DNA damage and cytochrome oxidase system dysfunction; hypoxanthine conversion to xanthine and xanthine conversion to uric acid catalyzed by xanthine oxidase; increases in the levels of cytokines, such as tumor necrosis factor and interleukin-1, during the surgical procedure and following neutrophil and macrophage stimulation, causing a respiratory burst; and oxygen-derived free radicals induced by catecholamine release during the perioperative period. In the present study, it was found that the levels of free radical indicators (superoxide anion, hydrogen peroxide and hydroxyl radical) were markedly increased at $2 \mathrm{~h}$ after surgery and decreased at $24 \mathrm{~h}$ after surgery, suggesting that surgery induced oxidative stress and increased the levels of free radicals. We therefore speculated that the respiratory burst was the main mechanism of oxidative stress.

Kang et al (5) found that midazolam exhibited antioxidant properties, but more recent studies have demonstrated that the clinical concentration of midazolam does not have a free radical-scavenging capacity (13). Previous studies investigating 
Table IV. Levels of SOD, GSH-Px and CAT.

\begin{tabular}{lccc}
\hline Parameter & T0 & T1 & T2 \\
\hline SOD $(U / m l)$ & & $31.5 \pm 7.3^{\mathrm{a}}$ & $35.3 \pm 5.2$ \\
M group & $43.4 \pm 7.3$ & $39.2 \pm 7.5^{\mathrm{a}, \mathrm{c}}$ & $45.1 \pm 5.7^{\mathrm{b}, \mathrm{c}}$ \\
P group & $46.5 \pm 7.1$ & $37.9 \pm 3.9^{\mathrm{a}, \mathrm{c}}$ & $44.4 \pm 5.5^{\mathrm{b}, \mathrm{c}}$ \\
D group & $47.1 \pm 4.7$ & & $23.5 \pm 6.1$ \\
GSH-Px U & & $19.5 \pm 6.0^{\mathrm{a}}$ & $33.3 \pm 9.6^{\mathrm{b}, \mathrm{c}}$ \\
M group & $37.8 \pm 8.6$ & $27.3 \pm 8.9^{\mathrm{a}, \mathrm{c}}$ & $38.1 \pm 8.2^{\mathrm{b}, \mathrm{c}}$ \\
P group & $39.8 \pm 9.4$ & $26.1 \pm 7.8^{\mathrm{a}, \mathrm{c}}$ & \\
D group & $38.0 \pm 10.5$ & & $6.3 \pm 1.0$ \\
CAT U/ml & & $5.9 \pm 1.1$ & $6.2 \pm 1.1$ \\
M group & $6.3 \pm 1.2$ & $6.0 \pm 1.3$ & $6.2 \pm 1.2$ \\
P group & $6.4 \pm 1.0$ & $6.1 \pm 1.2$ & \\
D group & $6.2 \pm 0.9$ & $P<0.05 . \mathrm{Re}$ \\
\hline
\end{tabular}

${ }^{\mathrm{a} C}$ Compared with T0, $\mathrm{P}<0.05$; ${ }^{\mathrm{b}}$ compared with $\mathrm{T} 1, \mathrm{P}<0.05$; ${ }^{\mathrm{c}}$ compared with $\mathrm{M}$ group, $\mathrm{P}<0.05$. Results are presented as the mean \pm standard deviation. M group, midazolam group; P group, propofol group; D group, dexmedetomidine group; SOD, superoxide dismutase; GSH-Px, glutathione peroxidase; CAT, catalase.

Table V. Frequencies of micronuclei, nucleoplasmic bridges and nuclear buds in 1,000 cells.

\begin{tabular}{lccc}
\hline Parameter & T0 & T1 & \\
\hline Micronuclei & & & \\
M group & $19.3 \pm 4.8$ & $41.5 \pm 6.3^{\mathrm{a}}$ & $30.3 \pm 4.2^{\mathrm{b}}$ \\
P group & $17.0 \pm 3.0$ & $36.2 \pm 4.2^{\mathrm{a}, \mathrm{c}}$ & $25.1 \pm 3.3^{\mathrm{b}, \mathrm{c}}$ \\
D group & $18.4 \pm 4.6$ & $35.9 \pm 3.3^{\mathrm{a}, \mathrm{c}}$ & $24.4 \pm 3.2^{\mathrm{b}, \mathrm{c}}$ \\
Nucleoplasmic bridges & & & $7.6 \pm 4.3^{\mathrm{b}}$ \\
M group & $7.3 \pm 2.3$ & $9.8 \pm 2.5^{\mathrm{a}}$ & $6.8 \pm 4.0^{\mathrm{b}}$ \\
P group & $6.8 \pm 3.1$ & $8.8 \pm 2.3^{\mathrm{a}}$ & $7.3 \pm 4.2^{\mathrm{b}}$ \\
D group & $7.9 \pm 3.2$ & $9.3 \pm 2.2^{\mathrm{a}}$ & \\
Nuclear buds & & & $6.2 \pm 3.3$ \\
M group & $5.6 \pm 2.3$ & $6.4 \pm 2.8$ & $5.8 \pm 2.1$ \\
P group & $5.3 \pm 3.0$ & $6.3 \pm 2.7$ & $5.9 \pm 1.2$ \\
D group & $5.9 \pm 2.1$ & & \\
\hline
\end{tabular}

${ }^{\mathrm{a} C}$ Compared with T0, $\mathrm{P}<0.05$; ${ }^{\mathrm{b}}$ compared with $\mathrm{T} 1, \mathrm{P}<0.05$; ${ }^{\mathrm{c}}$ compared with $\mathrm{M}$ group, $\mathrm{P}<0.05$ (Mann-Whitney U test). Results are presented as the mean \pm standard deviation. M group, midazolam group; $\mathrm{P}$ group, propofol group; D group, dexmedetomidine group.

the effects of sedatives on free radicals have shown that propofol can not only directly react with free radicals (14), but also enhance heme oxygenase expression and generate antioxidant activities (15), as its molecular structure is similar to that of the endogenous antioxidant vitamin E. Nishina et al (16) found that the clinical concentration of dexmedetomidine had no effect on the chemotaxis and phagocytosis of neutrophils and the production of the superoxide anion; however, Taniguchi et al (17) suggested that dexmedetomidine could reduce the inflammatory cell response and inhibit the release of pro-inflammatory cytokines and the generation of oxygen free radicals. In the present study it was found that the levels of superoxide anion, hydrogen peroxide and hydroxyl radical in the $\mathrm{P}$ and $\mathrm{D}$ groups were lower $2 \mathrm{~h}$ after the surgery than those in the M group, suggesting that propofol and dexmedetomidine are stronger radical scavengers.

MDA, which is a product of lipid peroxidation, is the most commonly used indicator of oxidative stress. Lipid peroxidation, which is the primary mechanism by which free radicals induce cellular damage, can cause decreases in membrane fluidity and permeability, and membrane protein denaturation. Hydroxyl radicals induce the peroxidation of unsaturated fatty acids and form a lipid peroxidation chain reaction (18); only when two lipid radicals react to form a non-radical product or when the radicals are quenched by an antioxidant molecule, such as $\alpha$-tocopherol (vitamin E), is the chain reaction terminated (19). Propofol can reduce lipid peroxidation due to its similar molecular structure to vitamin $\mathrm{E}$ (20). The present results also showed that the levels 
of MDA in the $\mathrm{P}$ and $\mathrm{D}$ groups at $2 \mathrm{~h}$ after surgery were lower than those in the $\mathrm{M}$ group, demonstrating that propofol and dexmedetomidine can significantly reduce lipid peroxidation during surgery when compared with midazolam. At $24 \mathrm{~h}$ after surgery, the level of MDA in the D group was the lowest among the three groups, suggesting that dexmedetomidine exerts the strongest protection against lipid peroxidation; however, the mechanism has yet to be elucidated.

Surgical stress can cause a decrease in the antioxidant enzyme activities of the body (21). A previous study showed that propofol and dexmedetomidine could enhance the SOD activity of human blood, with similar effects exhibited by both drugs (22). Another investigation found that propofol was a more potent enhancer of SOD activity than midazolam (23). In the present study, the levels of SOD and GSH-Px in the M group at $2 \mathrm{~h}$ after surgery were markedly lower than those in the $\mathrm{P}$ and D groups, suggesting that surgical trauma would decrease the activities of SOD and GSH-Px and that propofol and dexmedetomidine could enhance the activities of the two indicators.

The levels of CAT did not show any differences among the groups, indicating that surgical trauma and increased free radical production have few effects on the level of CAT. This may be a result of GSH-Px replacing the role of CAT in higher organisms, and particularly in humans (24).

It has previously been shown that the active metabolites (active oxygen ions, peroxides, free radicals and other ROS materials) generated by oxidative stress attack the body and lead to an increase in the micronucleus frequency (25). In the present study, therefore, the CBMN assay was used to assess the oxidative DNA damage of the peripheral blood lymphocytes of the patients, and to further to evaluate the effects of the three sedatives. It was found that the micronucleus and NPB frequencies were significantly higher at $\mathrm{T} 1$ than those at $\mathrm{T} 0$, and were lower at T2 than that at T1, showing that the levels of oxidative DNA damage were higher following surgery but decreased with time. Furthermore, it was found that the micronucleus frequencies of the P and D groups at T1 and T2 were lower than the frequency of the $\mathrm{M}$ group, suggesting that propofol and dexmedetomidine had a superior antioxidant function. Since it is the NPBs, not the NBUDs, that eventually form MN (25), no significant differences were found in the frequency of NBUDs.

In conclusion, the administration of propofol or dexmedetomidine leads to lower levels of oxidative stress and more desirable effects on the antioxidant system following surgery than midazolam. Furthermore, dexmedetomidine exerts longer-acting antioxidant effects than propofol; however, the effects of sedatives on the antioxidant system, treatment of diseases and disease outcome require further clinical studies.

\section{Acknowledgements}

This study was supported in part by the Medical Research Project of the Health Department of Jiangsu Province (no. Z201218) and the Six Talent Peaks Program of Jiangsu Province (no. WSN-024, 2013).

\section{References}

1. Møller P, Loft S, Lundby C and Olsen NV: Acute hypoxia and hypoxic exercise induce DNA strand breaks and oxidative DNA damage in humans. FASEB J 15: 1181-1186, 2001.
2. Fenech M: Cytokinesis-block micronucleus cytome assay. Nat Protoc 2: 1084-1104, 2007.

3. Collier BR, Giladi A, Dossett LA, Dyer L, Fleming SB and Cotton BA: Impact of high-dose antioxidants on outcomes in acutely injured patients. JPEN J Parenter Enteral Nutr 32: 384-388, 2008.

4. Christman JW, Blackwell TS and Juurlink BH: Redox regulation of nuclear factor kappa B: therapeutic potential for attenuating inflammatory responses. Brain Pathol 10: 153-162, 2000.

5. Kang MY, Tsuchiya M, Packer L and Manabe M: In vitro study on antioxidant potential of various drugs used in the perioperative period. Acta Anaesthesiol Scand 42: 4-12, 1998.

6. Rahman I and MacNee W: Lung glutathione and oxidative stress: implications in cigarette smoke-induced airway disease. Am J Physiol 277: 1067-1088, 1999.

7. Rahman I and MacNee W: Role of transcription factors in inflammatory lung diseases. Thorax 53: 601-612, 1998.

8. Biernacki M,Bigda J, Jankowski K, Wozniak M and Sledziński Z: Increased serum levels of markers of oxidative stress during kidney transplantation. Transplant Proc 34: 544-555, 2002.

9. Matata BM and Galiñanes M: Cardiopulmonary bypass exacerbates oxidative stress but does not increase proinflammatory cytokine release in patients with diabetes compared with patients without diabetes: Regulatory effects of exogenous nitric oxide. J Thorac Cardiovasc Surg 120: 1-11, 2000.

10. Tsukioka T, Takemura S, Minamiyama Y, et al: Local and systemic impacts of pleural oxygen exposure in thoracotomy. Biofactors 30: 117-128, 2007.

11. Sun G, Hu W, Zhang J and Lu Y: The nature of manganese superoxide dismutase expression in esophageal cancer cell. Int Med J 20: 711-715, 2013.

12. Sun G, Hu W, Wang Y and Li C: The effect of MnSOD overexpression and BSO on radiosensitivity in esophageal cancer cells. Int Med J 20: 567-570, 2013.

13. Hata M, Kobayashi K, Yoshino F, et al: Direct assessment of the antioxidant properties of midazolam by electron spin resonance spectroscopy. J Anesth 25: 765-769, 2011.

14. Murphy PG, Myers DS, Davies MJ, Webster NR and Jones JG: The antioxidant potential of propofol (2,6-diisopropylphenol). $\mathrm{Br}$ J Anaesth 68: 613-618, 1992.

15. Liang C, Cang J, Wang H and Xue Z: Propofol attenuates cerebral ischemia/reperfusion injury partially using heme oxygenase-1. J Neurosurg Anesthesiol 25: 311-316, 2013.

16. Nishina K, Akamatsu H, Mikawa K, Shiga M, Maekawa N, Obara $\mathrm{H}$ and Niwa $\mathrm{Y}$ : The effects of clonidine and dexmedetomidine on human neutrophil functions. Anesth Analg 88: 452-458, 1999.

17. Taniguchi T, Kurita A, Kobayashi K, Yamamoto K and Inaba H: Dose- and time-related effects of dexmedetomidine on mortality and inflammatory responses to endotoxin-induced shock in rats. J Anesth 22: 221-228, 2008.

18. Thorburne SK and Juurlink BH: Low glutathione and high iron govern the susceptibility of oligodendroglial precursors to oxidative stress. J Neurochem 67: 1014-1022, 1996.

19. Halliwell B: Oxygen radicals as key mediators in neurological disease: Fact or fiction? Ann Neurol 32 Suppl: S10-S15, 1992.

20. Murphy PG, Bennett JR, Myers DS, Davies MJ and Jones JG. The effect of propofol anaesthesia on free radical-induced lipid peroxidation in rat liver microsomes. Eur J Anaesthesiol 10: 261-266, 1993.

21. Keller GA, Barke R, Harty JT, Humphrey E and Simmons RL: Decreased hepatic glutathione levels in septic shock. Predisposition of hepatocytes to oxidative stress: An experimental approach. Arch Surg 120: 941-945, 1985.

22. Dülger H, Kelemençe H, Göktaş U, Şekeroğlu MR, Katı I, Özcan S and Baran FC: The effects of dexmedetomidine and propofol on the antioxidant system. Eur J Basic Med Sci 1: 21-27, 2011.

23. Xia WF, Liu Y, Zhou QS, Tang QZ and Zou HD: Comparison of the effects of propofol and midazolam on inflammation and oxidase stress in children with congenital heart disease undergoing cardiac surgery. Yonsei Med J 52: 326-332, 2011.

24. Choi KM, Kang CM, Cho ES, Kang SM, Lee SB and Um HD. Ionizing radiation-induced micronucleus formation is mediated by reactive oxygen species that are produced in a manner dependent on mitochondria, Nox1, and JNK. Oncol Rep 17: 1183-1188, 2007.

25. Rao X, Zhang Y, Yi Q, Hou H, Xu B, Chu L, et al: Multiple origins of spontaneously arising micronuclei in HeLa cells: Direct evidence from long-term live cell imaging. Mutat Res 646: 41-49, 2008. 